\title{
Metriplectic Algebra for Dissipative Fluids in Lagrangian Formulation
}

\author{
Massimo Materassi ${ }^{1}$ * \\ 1 Istituto dei Sistemi Complessi ISC-CNR, via Madonna del Piano 10, 50019 Sesto Fiorentino \\ (Florence), Italy; E-Mails: massimo.materassi@isc.cnr.it, massimomaterassi27@gmail.com
}

* Author to whom correspondence should be addressed; E-Mail: massimo.materassi@ isc.cnr.it (F.L.); Tel.: +39-055-5226627; Fax: +39-055-5226688.

Received: 10 September October 2014 / Accepted: 15 October 2014 / Published: 3 November 2014

\begin{abstract}
The dynamics of dissipative fluids in Eulerian variables may be derived from an algebra of Leibniz brackets of observables, the metriplectic algebra, that extends the Poisson algebra of the frictionless limit of the sytem via a symmetric semidefinite component, that generates dissipative forces. The metriplectic algebra includes the conserved total Hamiltonian $H$, generating the non-dissipative part of dynamics, and the entropy $S$ of those microscopic degrees of freedom draining energy irreversibly, that generates dissipation. This $S$ is a Casimir invariant of the Poisson algebra to which the metriplectic algebra reduces in the frictionless limit. The role of $S$ is as paramount as that of $H$, but this fact may be underestimated in the Eulerian formulation because $S$ is not the only Casimir of the symplectic non-canonical part of the algebra. Instead, when the dynamics of the non-ideal fluid is written through the parcel variables of the Lagrangian formulation, the fact that entropy is symplectically invariant clearly appears to be related to its the microscopic degrees of freedom of the fluid, that do not participate at all to the symplectic canonical part of the algebra (which, indeed, involves and evolves only the macroscopic degrees of freedom of the fluid parcel).
\end{abstract}

Keywords: Fluid dynamics; Hamiltonian formulations; Lagrangian and Hamiltonian mechanics

PACS Codes: 47.10.A, 47.10.Df, 47.65.Md, 45.20.Jj. 


\section{Introduction}

The history of Theoretical Physics is, to a certain extent, that of the discovery of symmetries of physical laws, allowing to bypass the necessity of solving the equations of motion explicitly and gaining deep insights about the essence of first principles themselves.

The highest achievements of this simplification process are the least action principles [1, 2], with the Feynman path integral [3] as their most recent descendant, and the study of invariances [4]; the Hamiltonian formalism [2, 5] and the Hamilton-Jacobi theory [5, 6]. In the context of Hamiltonian mechanics, the dynamics of physical systems appears in the form of an algebra of Poisson brackets [7], composing together the physical observables to both represent the motion of the system and the symmetry properties of its dynamics. This route to the algebrization of dynamics also leads to Dirac's formulation of Quantum Mechancis [8], according to which the algebra of quantum observables is simply a commutation algebra of operators, isomorphic to the Poisson algebra of the respective classical ones.

However, almost all the benefits of the just mentioned development are, generally speaking, restricted to the Physics of non dissipative systems: in Lagrangian and Hamiltonian mechanics, as well as in the context of action principles [5,7], only systems undergoing conservative forces are treated, while no form of "dissipation" is considered in the fundamental quantum laws, intended as the basic principles of Physics [9]. When dissipative quantum systems are referred to, one typically considers open systems in interaction with some "environment" only partially observed [10], and these are not regarded as "fundamental" (one should however mention the "line of thought", expressed in [11], for instance, in which dissipation is included in the fundamental laws of Quantum Mechanics).

A fruitful attempt to put dissipative systems on the way to algebrization of Physics is represented by the use of Leibniz algebræ $[12,13]$ generalizing the symplectic product. The dynamics of energetically closed systems relaxing to asymptotic equilibria due to dissipation has been described in this way by Morrison in [14] via the so called metriplectic formalism. The non-dissipative limit of the system is Hamiltonian, so that there exists some function $H$ and an algebra of Poisson brackets that describes the system in the absence of dissipation. When dissipation is turned on, the Hamiltonian is still constant during the motion, but friction drives the system to an asymptotic equilibrium: this is done generalizing the Poisson bracket so to include a symmetric semidefinite component, referred to as metric bracket. Then, dissipative processes are generated through that symmetric extension by an observable representing the entropy $S$ of the closure of the system.

Quite a few dynamical systems have been reformulated as metriplectic: in [14] the kinetic VlasovPoisson approximation of a collisional plasma was described as Hamiltonian in the collisionless limit, while collisional terms are shown to arise from a metric bracket. In [15] a non-ideal fluid described in Eulerian variables (EV) is presented as a non-canonical Hamiltonian system, with the addition of a metric bracket providing the dissipative terms due to the finite viscosity and thermal conductivity. In [16] the non-canonical Hamiltonian dynamics of a free rigid body throughout the space of its angular velocity is enriched by a metric contribution through which the rotator is made relax down to asymptotic equilibria at which the system spins around one of its inertial axes. In the same work, the dissipative Vlasov-Poisson equation is examined again. 
A general review of Poisson and metric brackets to describe energetically isolated or non-isolated systems (referred to as complete and incomplete) may be found in [17].

In his PhD thesis [18], Fish examines metriplectic systems of various types under the point of view of manifold properties, and also gives interesting examples from applied physics and biophysics.

The metriplectic system describing neutral fluids in [15] has been generalized to non-ideal magnetohydrodynamics in [19], while examples of how to algebrize simple mechanical systems with friction are provided in [20].

In the present paper, the Lagrangian Formulation $(L F)$ of the metriplectic algebra for a viscous fluid is constructed. The symplectic part of the metriplectic system is taken from [15, 21, 22], while the metric part is an original contribution presented here for the first time, as far as the author is aware of, by mapping the metric bracket in EV to its expression in parcel variables.

Even if rather interesting from the viewpoint of mathematical completeness, still the translation of the Eulerian metriplectic algebra to the Lagrangian one can be questioned to be worth the effort in physical terms. Instead, it should be underlined that the symmetry-related role of the fluid entropy appears much clearer in the Lagrangian algebra than in the Eulerian one, not to mention that whenever the use of LF is preferred to that of EF, the expressions found here will be applied.

The fluid entropy has zero Poisson bracket with any other quantity in both formulations, but the expression of the symplectic product in Lagrangian variables (LV) makes it clear that $S$ is not a Casimir invariant due to the parcel relabeling symmetry (that allows the fluid to possess an Eulerian representation at all), but simply because it encodes of degrees of freedom involved in parcel dynamics only through dissipation.

In Section 2 the general framework of metriplectic complete systems is sketched, while in Section 3 we discuss briefly the role of Casimir invariants of the theory with respect to algebra reduction and dissipative processes.

Section 4 is dedicated to the key result of this paper: the Lagrangian Formulation is constructed for viscous fluids, and their metriplectic algebra is formulated in the material variables. With this result in mind, a speculation on the nature of the fluid entropy as a Casimir invariant of the theory is presented in Section 5.

Conclusions are reported in Section 6, where possible applications and future developments of the present research are also sketched.

\section{Metriplectic complete systems}

Consider an energetically closed system with dissipation, and describe its state as a point $\psi$ moving in a suitable phase space $\mathbf{V}$. Also, refer to its algebra of observables $O$ as a subset of $C^{\infty}(\mathbf{V}, \mathbf{R})$.

According to the metriplectic scheme, its dynamics $\dot{\psi}$ will be expressed as the sum of a nondissipative part $\dot{\psi}_{\text {non-diss }}=\{\psi, H\}$, generated by the Hamiltonian $H \in O$ through a Poisson bracket structure, and the dissipative part $\dot{\psi}_{\text {diss }}=\lambda(\psi, S)$, where (.,.) is a symmetric semidefinite Leibniz bracket

$$
(A, B)=(B, A), \quad(A, A) \leq 0 \quad \forall \quad A, B \in O,
$$


referred to as metric bracket, and $\lambda$ is a negative constant parameter (making physical sense only in the correspondence of the asymptotic equilibrim [19]).

The generator $S$ of the dissipative dynamics $\dot{\psi}_{\text {diss }}$ has zero Poisson bracket with any other observable depending on $\psi$

$$
\{S, A\}=0 \quad \forall \quad A \in O,
$$

while the metric bracket (., .) must have $H$ among its null modes

$$
(H, A)=0 \quad \forall \quad A \in O .
$$

If the evolution of the system works as

$$
\dot{\psi}=\dot{\psi}_{\text {non-diss }}+\dot{\psi}_{\text {diss }}=\{\psi, H\}+\lambda(\psi, S),
$$

then any quantity $\Phi \in O$ depending on $\psi$ evolves according to the same rule

$$
\dot{\Phi}(\psi)=\{\Phi(\psi), H\}+\lambda(\Phi(\psi), S) .
$$

Due to the conditions (1) and (2), this general rule also implies

$$
\dot{H}=0, \quad \dot{S}=\lambda(S, S) \geq 0 \text { : }
$$

the first of these equations means that $H$ is constant because it is not altered by dissipation, that just redistributes energy but does not destroy it; the second condition in (5) states that $S$ asymptotically and monotonically grows during the motion, as a Lyapunov quantity is expected to do in the correspondence of an asymptotic stable state [23].

The conditions (1) and (2), together with the properties of $\{.,$.$\} and (., .) as Leibniz brackets [12],$ allow for the definition of a total metriplectic generator $F=H+\lambda S$ so that, provided the new bracket

$$
\langle\langle A, B\rangle\rangle=\{A, B\}+(A, B)
$$

is defined, one may simply state

$$
\dot{\psi}=\langle\langle\psi, F\rangle\rangle,
$$

and $\dot{\Phi}=\langle\langle\Phi, F\rangle\rangle$ for any observable $\Phi$. The new Leibniz structure defined in (6) is the metriplectic bracket, while the metriplectic generator $F$ is sometimes referred to as free energy.

\section{Casimir invariants}

The condition (1) attributes to the metric generator $S$, whatever it is physically, the algebraic character of Casimir invariant (CI) of the Poisson bracket $\{.,$.$\} .$

Now, the metric generator may be a CI for one of the two following reasons.

Either, the symplectic bracket $\{.,$.$\} includes derivatives with respect to the variables on which S$ depends too, and nevertheless admits a non-trivial kernel to which $S$ belongs; this case, we will refer to as $\mathrm{C} 1$, is typical for Poisson algebræ $\left(A_{\text {red }},\{., .\}_{\text {red }}\right)$ obtained by reducing some Poisson algebra $(A,\{. .,\}$. to the algebra of all the observables invariant under a certain group of transformations $\mathbf{G}$ : if $g \in A$ is a symplectic generator of those transformations, clearly $\{\Phi, g\}_{\text {red }}=0$ for any element $\Phi \in A_{\text {red }}, g$ is a CI for the bracket $\{., .\}_{\text {red }}$. 
Or, the variables forming $S$ do not appear at all in the definition of the Poisson bracket, so that $S$ belongs to the kernel of it as does any variable outside the system; this other case, referred to as $\mathrm{C} 2$, is that of a Poisson algebra $\left(A_{0},\{., .\}_{0}\right)$ describing a system of variables $\psi_{0}$ in interaction with some environment, of which an effective description is given via a variable $z$ external to the system: then, any $C(z)$ is trivially a CI of $\{.,\}_{0}$, since the latter depends only on derivatives with respect to $\psi_{0}$ but does not involve any derivative in $z$. A metriplectic system describing the relaxation of "macroscopic" variables $\psi_{0}$ due to the interaction with some microscopic degrees of freedom ( $\left.\mu \mathrm{DoF}\right)$ may be conceived by defining a metric bracket "driven" by $C(z)$ and acting on the "total" state $\psi \stackrel{\text { def }}{=}\left(\psi_{0}, z\right)$, where $z$ is a coarse grained description of the $\mu \mathrm{DoF}$.

Throughout the literature mentioned in Section 1, one meets examples of both kinds C1 and C2.

The free dumped rotator presented in [16], and revisited in [18] is easily recognized to be a C1 case: the square angular momentum is such a CI when the phase space of the rotator is reduced from the 6 dimensional space of angles and their canonical momenta to the $\mathbf{R}^{3}$ of angular velocities. Systems with dissipative constants regarded as control parameters depending on an external variable are properly $\mathrm{C} 2$ cases (e.g., the Lodka-Volterra, Lorentz and Van Der-Pole systems in [18], or the elementary mechanics dissipative systems reported in [20], where the external variable is the state of a thermal bath). Last but not least, deciding whether the Boltzmann entropy playing the role of the metric generator for the Vlasov-Poisson collisional plasma is a $\mathrm{C} 1$ or $\mathrm{C} 2$ quantity deserves a deeper investigation, involving the fact that Vlasov-Poisson equation results from the truncation of a hierarchy of equations involving many-particle variables [24], the symplectic limit of which has been studied in [25]. The origin of being a CI for the entropy of a viscous fluid is investigated here, writing its metriplectic algebra explicitly in LV, as done in Section 4 below.

\section{Lagrangian Formulation for viscous fluids}

In [15] the viscous fluid equation is described in the Eulerian Formalism (EF), via the fields mass density $\rho(\vec{x}, t)$, velocity $\vec{v}(\vec{x}, t)$ and mass-specific entropy density $\sigma(\vec{x}, t)$. In the non-dissipative limit the dynamics takes a non-canonical Hamiltonian form: the Poisson bracket between two any functionals $\Phi[\rho, \vec{v}, \sigma]$ and $\Psi[\rho, \vec{v}, \sigma]$ is defined as

$$
\begin{aligned}
& \{\Phi, \Psi\}_{E}=-\int_{\mathbf{R}^{3}} d^{3} x\left[\frac{\delta \Phi}{\delta \rho} \partial_{\alpha}\left(\frac{\delta \Psi}{\delta v_{\alpha}}\right)+\frac{\delta \Psi}{\delta \rho} \partial_{\alpha}\left(\frac{\delta \Phi}{\delta v_{\alpha}}\right)-\frac{1}{\rho} \frac{\delta \Psi}{\delta v_{\alpha}} \varepsilon_{\alpha \gamma \beta} \varepsilon^{\beta \delta \eta} \frac{\delta \Psi}{\delta v_{\gamma}} \partial_{\delta} v_{\eta}+\right. \\
& \left.+\frac{1}{\rho} \partial_{\alpha} \sigma\left(\frac{\delta \Phi}{\delta \rho} \frac{\delta \Psi}{\delta v_{\alpha}}-\frac{\delta \Psi}{\delta \rho} \frac{\delta \Phi}{\delta v_{\alpha}}\right)\right]
\end{aligned}
$$

where Greek indices are used for the $S O(3)$-vector components of $\vec{v}$ and of the position $\vec{x}$ in the space, and a summation convention holds, so that scalar products in $\mathbf{R}^{3}$ read $\vec{v} \cdot \vec{w}=v^{\alpha} w_{\alpha}$. The symbol $\partial_{\alpha}=\frac{\partial}{\partial x^{\alpha}}$ is used for spatial gradients.

The Hamiltonian functional of the system reads

$$
H[\rho, \vec{v}, \sigma]=\int_{\mathbf{R}^{3}} d^{3} x\left[\frac{\rho v^{2}}{2}+\rho U(\rho, \sigma)+\rho \phi\right],
$$


where $\rho U d^{3} x$ is the amount of internal energy attributed to the infinitesimal volume $d^{3} x$ around the position $\vec{x} . \phi$ is an external potential.

$H$ generates the motion of any observable $\Phi[\rho, \vec{v}, \sigma]$ as $\dot{\Phi}=\{\Phi, H\}$ thanks to the Poisson bracket (7): the non-dissipative Navier-Stokes equations

$$
\left\{\begin{array}{l}
\partial_{t} v_{\alpha}=-v_{\beta} \partial^{\beta} v_{\alpha}-\frac{1}{\rho} \partial_{\alpha} p-\partial_{\alpha} \phi \\
\partial_{t} \rho=-\partial^{\beta}\left(\rho v_{\beta}\right) \\
\partial_{t} \sigma=-v_{\beta} \partial^{\beta} \sigma
\end{array}\right.
$$

where $p$ is the pressure, hence follow.

Let then viscosity and thermal conductivity be finite.

Let the viscosity tensor be of the form $\Sigma^{\alpha \beta}=\Lambda^{\alpha \beta \gamma \delta} \partial_{\gamma} v_{\delta}$, with $\Lambda$ constant (this $\Lambda$ is formed by Kronecker tensors and physical "constitutive" constants, see [19]); let the heat flux $\vec{I}$ be related to the local temperature $T$ as $I_{\alpha}=-\kappa \partial_{\alpha} T$ : then, the symplectic algebra (7) must be completed by the metric bracket

$$
\begin{aligned}
& (\Phi, \Psi)_{E}=\frac{1}{\lambda} \int_{\mathbf{R}^{3}} d^{3} x\left\{T \Lambda_{\alpha \beta \gamma \delta}\left[\partial^{\alpha}\left(\frac{1}{\rho} \frac{\delta \Phi}{\delta v_{\beta}}\right)-\frac{1}{\rho T} \partial^{\alpha} v^{\beta} \frac{\delta \Phi}{\delta \sigma}\right]\left[\partial^{\gamma}\left(\frac{1}{\rho} \frac{\delta \Psi}{\delta v_{\delta}}\right)-\frac{1}{\rho T} \partial^{\gamma} v^{\delta} \frac{\delta \Psi}{\delta \sigma}\right]+\right. \\
& \left.+\kappa T^{2} \partial^{\alpha}\left(\frac{1}{\rho T} \frac{\delta \Phi}{\delta \sigma}\right) \partial_{\alpha}\left(\frac{1}{\rho T} \frac{\delta \Psi}{\delta \sigma}\right)\right\}
\end{aligned}
$$

so that, given the total entropy of the fluid as

$$
S[\rho, \sigma]=\int_{\mathbf{R}^{3}} \rho \sigma d^{3} x
$$

the dynamics reads

$$
\dot{\Phi}=\{\Phi, H\}_{E}+\lambda(\Phi, S)_{E}
$$

This gives rise to the non-ideal equations of motion:

$$
\left\{\begin{array}{l}
\partial_{t} v_{\alpha}=-v_{\beta} \partial^{\beta} v_{\alpha}-\frac{1}{\rho} \partial_{\alpha} p-\partial_{\alpha} \phi+\frac{1}{\rho} \partial^{\kappa}\left(\Lambda_{\kappa \alpha \beta \gamma} \partial^{\beta} v^{\gamma}\right), \\
\partial_{t} \rho=-\partial^{\beta}\left(\rho v_{\beta}\right), \\
\partial_{t} \sigma=-v_{\beta} \partial^{\beta} \sigma+\frac{1}{\rho T} \Lambda_{\kappa \alpha \beta \gamma} \partial^{\kappa} v^{\alpha} \partial^{\beta} v^{\gamma}+\frac{\kappa}{\rho T} \partial^{2} T .
\end{array}\right.
$$

The symbol $\partial^{2}=\partial^{\beta} \partial_{\beta}$ has been used.

In the LF, the fluid is subdivided into material parcels labeled by a continuous three-index $\vec{a}$, and the motion and evolution of each $\vec{a}$-th parcel is followed [26]. As far as its motion throughout the space is concerned, the $\vec{a}$-th fluid parcel is described at time $t$ by its position $\vec{\zeta}(\vec{a}, t)$ and its momentum $\vec{\pi}(\vec{a}, t)$ (in order to give a more concrete sense to the label $\vec{a}$, the choice 


$$
\vec{a}=\vec{\zeta}(\vec{a}, 0)
$$

can be made). Since the parcel is a system of $\mathbf{O}\left(10^{23}\right)$ microscopic particles, it must be equipped also by some variable describing those $\mu \mathrm{DoF}$ : its mass-specific entropy density $s(\vec{a}, t)$ is given this role [27]. The fact that the $\mu \mathrm{DoF}$ of the $\vec{a}$-th parcel are all encoded in the thermodynamical variable $s(\vec{a}, t)$ suggests that they are treated statistically. In a sense, the metriplectic formalism is the algebrization of a stochastic dynamics in which what remains of the probabilistic noise is its equilibrim thermodynamics [20].

A vivid representation of the variables $(\vec{\zeta}, \vec{\pi}, s)$ may be that $(\vec{\zeta}, \vec{\pi})$ are the variables of the parcel's centre-of-mass, while $s$ encodes the thermodynamics of the relative variables [28].

The field configuration $(\vec{\zeta}, \vec{\pi}, s)$ represents the state of the fluid in LF, let's indicate its functional phase space as $\mathbf{V}_{L}$. In LF the hypothesis of parcel identity conservation is made: this means that at every time $t$ the map $\vec{a} \mapsto \vec{\zeta}(\vec{a}, t)$ is a diffeomorphism from the space initially occupied by the continuum $\mathbf{D}_{0}$ and the one it occupies at time $t, \mathbf{D}(t) \subseteq \mathbf{R}^{3}$. If its Jacobian matrix $J_{i}^{\mu}=\frac{\partial \zeta^{\mu}}{\partial a^{i}}$ is defined, with the volume expansion factor $\mathbf{J}=\operatorname{det} J$, then the measure of the infinitesimal volume $d^{3} \zeta(\vec{a}, t)$ of the $\vec{a}$-th parcel at time $t$ is related to its initial volume $d^{3} a$ by the law $d^{3} \zeta=\mathbf{J} d^{3} a$. Also, these diffeomorphisms show a (semi)-group property with respect to the parameter $t$ : $\vec{\zeta}\left(\vec{a}, t_{1}+t_{2}\right)=\vec{\zeta}\left(\vec{\zeta}\left(\vec{a}, t_{1}\right), t_{2}\right)$.

Vector components of $\vec{\zeta}$ and $\vec{\pi}$ are labeled by Greek indices, as $\vec{v}$ and $\vec{x}$ in the EF, while Latin indices label the components of $\vec{a}$ (even if $\vec{\zeta}$ and $\vec{a}$ belong to the same physical space, as shown in (11), we prefer to use different indices for components of dynamical variables and of the label $\vec{a}$ ).

The Hamiltonian (8) is easily re-written in the LF as

$$
H[\vec{\zeta}, \vec{\pi}, s]=\int_{\mathbf{D}_{0}} d^{3} a\left[\frac{\pi^{2}}{2 \rho_{0}}+\rho_{0} U\left(\frac{\rho_{0}}{\mathbf{J}}, s\right)+\rho_{0} \phi(\vec{\zeta})\right] .
$$

$\rho_{0}(\vec{a})$ is the initial mass density of the $\vec{a}$-th parcel. The mass-specific internal energy density $U$ depends on the density of the parcel, that reads $\rho=\frac{\rho_{0}}{\mathbf{J}}$ because of mass conservation [29], and on its entropy. The dynamics of the nondissipative limit in LV is governed by an apparently canonical Poisson bracket, reading:

$$
\{\Phi, \Psi\}_{L}=\int_{\mathbf{D}_{0}} d^{3} a\left[\frac{\delta \Phi}{\delta \zeta^{\alpha}(\vec{a})} \frac{\delta \Psi}{\delta \pi_{\alpha}(\vec{a})}-\frac{\delta \Psi}{\delta \zeta^{\alpha}(\vec{a})} \frac{\delta \Phi}{\delta \pi_{\alpha}(\vec{a})}\right]
$$

(the expression "apparently canonical" will be commented later on). For any physical observable $\Phi$ one has simply $\dot{\Phi}=\{\Phi, H\}_{L}$, giving rise to the equations of motion:

$$
\left\{\begin{array}{l}
\dot{\zeta}_{\alpha}=\pi_{\alpha}, \\
\dot{\pi}_{\alpha}=-\rho_{0} \frac{\partial \phi}{\partial \zeta^{\alpha}}+A_{\alpha}^{i} \frac{\partial}{\partial a^{i}}\left(\rho_{0} \frac{\partial U}{\partial \mathbf{J}}\right), \quad A_{\alpha}^{i}=\frac{\varepsilon_{\alpha \kappa \lambda} \varepsilon^{i m n}}{2} \frac{\partial \zeta^{\kappa}}{\partial a^{m}} \frac{\partial \zeta^{\lambda}}{\partial a^{n}} \\
\dot{s}=0
\end{array}\right.
$$


((14) the "dot" means "time derivative along the motion of the parcel", also called Lagrangian, or material, derivative).

In order to complete the dynamics of the non-ideal fluid in LF, the metric part must be produced. The first step is to consider that the equations of motion to be reproduced are the translation of the system (10) in parcel variables:

$$
\left\{\begin{array}{l}
\dot{\zeta}_{\alpha}=\pi_{\alpha}, \\
\dot{\pi}_{\alpha}=-\rho_{0} \frac{\partial \phi}{\partial \zeta^{\alpha}}+A_{\alpha}^{i} \frac{\partial}{\partial a^{i}}\left(\rho_{0} \frac{\partial U}{\partial \mathbf{J}}\right)+\Lambda_{\alpha \beta \gamma \delta} \mathbf{J} \nabla^{\beta} \nabla^{\gamma}\left(\frac{\pi^{\delta}}{\rho_{0}}\right), \\
\dot{s}=\frac{\mathbf{J}}{\rho_{0} T} \Lambda_{\alpha \beta \gamma \delta} \nabla^{\alpha}\left(\frac{\pi^{\beta}}{\rho_{0}}\right) \nabla^{\gamma}\left(\frac{\pi^{\delta}}{\rho_{0}}\right)+\frac{\kappa \mathbf{J}}{\rho_{0} T} \nabla^{\alpha} \nabla_{\alpha} T .
\end{array}\right.
$$

The definition of $A_{\alpha}^{i}$ was already given in (10). The operator $\nabla_{\mu}$ is the derivative with respect to $\zeta^{\mu}$ intended as the differential operator $\nabla_{\mu}=\frac{\partial a_{i}}{\partial \zeta^{\mu}} \frac{\partial}{\partial a_{i}}$, and it acts on $\vec{a}$-dependent fields through the chain rule; the operator $\nabla_{\mu}$ reads $\nabla_{\mu}=\left(J^{-1}\right)_{\mu i}(\partial \vec{\zeta})_{\frac{\partial}{\partial a_{i}}}$ in terms of the Jacobian $J(\partial \vec{\zeta})$. In (15) $T$ represents the temperature of the $\vec{a}$-th parcel.

The metric bracket $(.,)_{L}$ is obtained by requiring that it reproduces the equations (15) via the prescription

$$
\dot{\Phi}=\{\Phi, H\}_{L}+\lambda(\Phi, S)_{L}:
$$

in order to obtain it explicitly, one may consider $(\Phi, \Psi)_{E}$ in (9) and reason on the relationship between the parcel variables and the Eulerian fields

$$
\left\{\begin{array}{l}
\rho(\vec{x}, t)=\int_{\mathbf{D}_{0}} d^{3} a \rho_{0}(\vec{a}) \mathbf{J}(\partial \vec{\zeta}(\vec{a}, t)) \delta^{3}(\vec{\zeta}(\vec{a}, t)-\vec{x}), \\
\vec{v}(\vec{x}, t)=\int_{\mathbf{D}_{0}} d^{3} a \frac{\vec{\pi}(\vec{a}, t)}{\rho_{0}(\vec{a})} \delta^{3}(\vec{\zeta}(\vec{a}, t)-\vec{x}) \\
\sigma(\vec{x}, t)=\int_{\mathbf{D}_{0}} d^{3} a s(\vec{a}, t) \delta^{3}(\vec{\zeta}(\vec{a}, t)-\vec{x})
\end{array}\right.
$$

The Eulerian field is the value taken by the corresponding Lagrangian quantity attributed to the parcel that, at that given time, transits at that given point: hence, one should understand $\rho_{0}(\vec{a}) \mathbf{J}(\partial \vec{\zeta}(\vec{a}, t))$ in (9) in the place of $\rho(\vec{x}, t), \frac{\vec{\pi}(\vec{a}, t)}{\rho_{0}(\vec{a})}$ in the place of $\vec{v}(\vec{x}, t)$ and $s(\vec{a}, t)$ in the place of $\sigma(\vec{x}, t)$, provided the label $\vec{a}$ is chosen so that $\vec{\zeta}(\vec{a}, t)=\vec{x}$. The integral over $\mathbf{R}^{3}$ in $d^{3} x$ is replaced by an integral over $\mathbf{D}_{0}$ in $d^{3} \zeta=\mathbf{J} d^{3} a$.

Special care must be used to treat the relationship between the functional derivative with respect to any Eulerian field $\psi_{E}(\vec{x})$ and that with respect to the corresponding Lagrangian variable $\psi_{L}(\vec{a})$. These operations are in fact defined via Frechet derivatives 


$$
\left\{\begin{array}{l}
\frac{\delta \Phi}{\delta \psi_{E}(\vec{x})}=\lim _{\varepsilon \rightarrow 0} \frac{d}{d \varepsilon} \Phi\left[\psi_{E}(\vec{y})+\varepsilon \delta^{3}(\vec{y}-\vec{x})\right], \\
\frac{\delta \Phi}{\delta \psi_{L}(\vec{a})}=\lim _{\varepsilon \rightarrow 0} \frac{d}{d \varepsilon} \Phi\left[\psi_{L}(\vec{b})+\varepsilon \delta^{3}(\vec{b}-\vec{a})\right],
\end{array}\right.
$$

so that, even if $\psi_{E}$ and $\psi_{L}$ may be identified with each other, still the distributions $\delta^{3}(\vec{b}-\vec{a})$ and $\delta^{3}(\vec{y}-\vec{x})$, here to be understood as $\delta^{3}(\vec{\zeta}(\vec{b})-\vec{\zeta}(\vec{a}))$, do not exactly coincide: $\delta^{3}(\vec{b}-\vec{a})=\mathbf{J} \delta^{3}(\vec{\zeta}(\vec{b})-\vec{\zeta}(\vec{a}))$. As a result, one may write:

$$
\frac{\delta \Phi}{\delta \psi_{E}(\vec{\zeta}(\vec{a}))}=\frac{1}{\mathbf{J}(\partial \vec{\zeta}(\vec{a}))} \frac{\delta \Phi}{\delta \psi_{L}(\vec{a})} .
$$

All in all, the metric bracket for a viscous fluid in LF reproducing equations (15) with $S[s]$ as a metric generator reads:

$$
\begin{aligned}
& (\Phi, \Psi)_{L}=\frac{1}{\lambda} \int_{\mathbf{D}_{0}} \mathbf{J} d^{3} a\left\{T \Lambda_{\alpha \beta \gamma \delta}\left[\nabla^{\alpha}\left(\frac{\delta \Phi}{\delta \pi_{\beta}}\right)-\frac{1}{\rho_{0} T} \nabla^{\alpha}\left(\frac{\pi^{\beta}}{\rho_{0}}\right) \frac{\delta \Phi}{\delta s}\right]\left[\nabla^{\gamma}\left(\frac{\delta \Psi}{\delta \pi_{\delta}}\right)-\frac{1}{\rho_{0} T} \nabla^{\gamma}\left(\frac{\pi \delta}{\rho_{0}}\right) \frac{\delta \Psi}{\delta \sigma}\right]+\right. \\
& \left.+\kappa T^{2} \nabla^{\alpha}\left(\frac{1}{\rho_{0} T} \frac{\delta \Phi}{\delta s}\right) \nabla_{\alpha}\left(\frac{1}{\rho_{0} T} \frac{\delta \Psi}{\delta s}\right)\right\} .
\end{aligned}
$$

The bracket (17) is easily shown to exhibit all the necessary properties for it to be a metric bracket: it's thoroughly symmetric in the $\Phi \leftrightarrow \Psi$ exchange, while about semidefiniteness one may note

$$
(\Phi, \Psi)_{L}=(\Phi, \Psi)_{E}
$$

provided the correct "dictionary" is used, so that one may count of the fact that $(\Phi, \Psi)_{L}$ inherits all the good properties from those demonstrated for $(\Phi, \Psi)_{E}$ in $[15,19]$, and references therein.

With the finding (17) we have the complete metriplectic algebra of viscous fluid dynamics in the $\mathrm{LF}$, that can be reported as:

$$
\left\{\begin{array}{l}
\dot{\Phi}=\langle\langle\Phi, F\rangle\rangle_{L}, \\
\langle\langle\Phi, \Psi\rangle\rangle_{L}=\{\Phi, \Psi\}_{L}+(\Phi, \Psi)_{L}, \\
F=H+\lambda S \\
H=\int_{\mathbf{D}_{0}} d^{3} a\left[\frac{\pi^{2}}{2 \rho_{0}}+\rho_{0} U\left(\frac{\rho_{0}}{\mathbf{J}}, s\right)+\rho_{0} \phi(\vec{\zeta})\right], \quad S=\int_{\mathbf{D}_{0}} d^{3} a \rho_{0} s .
\end{array}\right.
$$

As anticipated before, the advantage of looking at the metriplectic fluid dynamics in the LF, instead of in the EF, is that a certain subtlety about entropy is clarified, that has to do with the question of it to be a CI of the theory.

\section{Entropy and the Casimir invariant condition}

Back to what described in Section 1, we speculate here on the entropy of fluids [15, 19], that appear as in-between the "two ways of being a Casimir" C1 and C2. 
On the one hand, this $S$ clearly encodes information on the $\mu \mathrm{DoF}$ of the continuum, while the fluid velocity describe a macroscopic point of view of the system, as it happens in the $\mathrm{C} 2$ case. On the other hand, Morrison and Padhye had algebraic reasons to show, in [21] and [22], that this $S$ belongs to a family of quantities conserved, via a "C1 mechanism", out of the reduction of the algebra (13) to the set $A_{E}$ of quantities $\Theta[\vec{\zeta}, \vec{\pi}, s]$ so that $\left\{\Theta, C_{1,2}\right\}_{L}=0$, that become the physical quantities in the EF, and are invariant under parcel relabeling transformations (RT) [29]. Examining the LF of the fluid, with the RT more clearly readable, the opinion of the author here has become that the viscous fluid may be considered on the same foot as those mentioned in [18] and [20], classifying its $S$ in the C2 case.

The RTs, on which the LF to EF reduction is based, are smooth invertible maps $\vec{a} \mapsto \vec{a}^{\prime}$ that leave the Hamiltonian (12) and the Eulerian fields (16) unchanged. The quantities acting as symplectic generators via the bracket $\{., .\}_{L}$ must belong to one of either the following families of functionals

$$
\left\{\begin{array}{l}
C_{1}[\vec{\zeta}, \vec{\pi}, s]=\int_{\mathbf{D}_{0}} d^{3} a \eta(\vec{a}) Q_{s}(\vec{a}), \quad Q_{s}(\vec{a})=\varepsilon^{i j k} \frac{\partial \pi^{\alpha}}{\partial a^{i}} \frac{\partial \zeta_{\alpha}}{\partial a^{j}} \frac{\partial s}{\partial a^{k}}, \\
C_{2}[s]=\int_{\mathbf{D}_{0}} d^{3} a W(\vec{a}) s(\vec{a}),
\end{array}\right.
$$

where $\eta(\vec{a})$ and $W(\vec{a})$ are arbitrary functions. The quantity $Q_{s}(\vec{a})$ is referred to as potential vorticity, while the entropy of the fluid is an example of $C_{2}[s]$, with $W(\vec{a})=\rho_{0}(\vec{a})$. Both $C_{1}$ and $C_{2}$ are in involution with any quantity in $A_{E}$, so that if the reduction with respect to the symmetry they generate is performed, they do become CI. The point is that, due to the fact that no derivative with respect to $s$ appears in $\{.,\}_{L}$, the quantities $C_{2}[s]$ are "already CI" in the symplectic algebra of the Lagrangian Formulation. Instead, a non-trivial set exists of LF functionals $\Xi[\vec{\zeta}, \vec{\pi}, s]$ so that $\left\{\Xi, C_{1}\right\}_{L} \neq 0$, with $C_{1}$ given in (18): this is the set of the quantities that can be constructed in the LF but that have not a corresponding Eulerian quantity, because they are not RT-invariant [21, 22, 29]. Moreover, the Poisson bracket $\{., .\}_{L}$ has been indicated as "apparently canonical" because, even if the Frechet derivatives $\frac{\delta}{\delta \zeta^{\alpha}(\vec{a})}$ and $\frac{\delta}{\delta \pi_{\alpha}(\vec{a})}$ in (13) appear just like they would be expected to in canonical brackets, still $s$ has no involvement in it, but is part of $\mathbf{V}_{L}$. This means that the symplectic operator giving rise to $\{.,\}_{L}$ is degenerate on $\mathbf{V}_{L}$, and the bracket is not "properly" canonical. It admits a nontrivial null space, the set of the quantities $C_{2}[s]$ in (18) of anything depending on $s$ only: we could visualize this by expressing the matrix related to $\{.,\}_{L}$ as

$$
\{\Phi, \Psi\}_{L}=\left(\partial_{\psi} \Phi\right)^{T} \cdot Z \cdot \partial_{\psi} \Psi, \quad Z=\left(\begin{array}{ccc}
0 & \mathbf{1}_{3} & 0 \\
-\mathbf{1}_{3} & 0 & 0 \\
0 & 0 & 0
\end{array}\right),
$$

being $\psi=(\vec{\zeta}, \vec{\pi}, s)$, while one also has $\mathbf{V}_{L}=\mathbf{R}^{6} \oplus \mathbf{R}$, being $\mathbf{R}^{6}$ that of the canonical variables $(\vec{\zeta}, \vec{\pi})$ and $\mathbf{R}$ that of $s$.

The physical difference between $S$ and any $C_{1}$ is that $S$ includes only the $\mu$ DoF responsible for dissipation, while the $C_{1}$ s mix them with the centre-of-mass variables $(\vec{\zeta}, \vec{\pi})$. In few words, only $S$ is expected to play the driving role in dissipation processes. 
The physical difference of roles for $C_{1}$ and $C_{2}$ in (18) persists in the metriplectic algebra of the fluid in the EF. In terms of Eulerian fields those quantities appear as follows

$$
\left\{\begin{array}{l}
C_{1 E}[\rho, \vec{v}, \sigma]=\int_{\mathbf{D}} d^{3} x \rho c_{1}\left(Q_{\sigma E}\right), \quad Q_{\sigma E}=\frac{1}{\rho} \vec{\partial} \sigma \cdot(\vec{\partial} \times \vec{v}), \\
C_{2 E}[\rho, \sigma]=\int_{\mathbf{D}} d^{3} x \rho c_{2}(\sigma)
\end{array}\right.
$$

(use has been made of the symbol $\vec{\partial}=\frac{\partial}{\partial \vec{x}}$ ): clearly, all the quantities $C_{1 E}$ or $C_{2 E}$ in (19) satisfy the prescription (1), so one could be tempted to generalize the expression of the free energy as

$$
\mathbf{F}=H+\lambda_{1} C_{1 E}+\lambda_{2} C_{2 E} ;
$$

the point is whether this gives rise to any sensible dynamics through the metriplectic algebra $\langle\langle., .\rangle\rangle_{E}=\{., .\}_{E}+(., .)_{E}$; for sure, as long as the metric bracket (9) is used, the equations of motion (10) are produced only choosing $\lambda_{1}=0$ and $C_{2 E}=S$, so that entropy seems to play a role that no other CI plays: assuming (10), the evolution of any $C_{k E}$ may be expressed as $\dot{C}_{k E}=\lambda\left(C_{k E}, S\right)_{E}$, for $k=1,2$. The Casimir $C_{1 E}$ instead does not generate any dynamics. Whether $\mathbf{F}$ in (20) may be "useful to dynamics" with symmetric brackets other than that in (9) remains an open question.

\section{Conclusions}

A viscous fluid with suitable border conditions relaxes to an asymptotic equilibrium due to the presence of dissipation, while it can be written in a Hamiltonian form in its frictionless limit. This is a perfect system to be put in a metriplectic form according to the prescriptions of [14], [20] and references therein.

Fluids may be represented in EF or in LF, and the metriplectic framework for the EF was already known [15]. Here, the metriplectic algebra in the LF is obtained, adopting the parcel variables as in [26] to describe the fluid in a metriplectic form: the resulting picture is rather clearer than the one in EF.

The position of the center-of-mass of the $\vec{a}$-th parcel $\vec{\zeta}(\vec{a})$ and its momentum $\vec{\pi}(\vec{a})$ undergo the dissipative interaction with the $\mu \mathrm{DoF}$ of the nearby parcels, encoded in the entropy of nearby parcels (of course, $\vec{\zeta}(\vec{a})$ and $\vec{\pi}(\vec{a})$ cannot interact directly with the $\mu \mathrm{DoF}$ of their own parcel, since no internal force can alter the motion of the centre-of-mass [30]). The novel result is the expression (17) of the metric bracket in parcel variables, through which the metric generator of dissipation, namely the fluid entropy $S$, makes viscosity act.

The pure Hamiltonian limit of the metriplectic system would actively involve only the variables $\vec{\zeta}(\vec{a})$ and $\vec{\pi}(\vec{a})$, as demonstrated by the expression (13), in which no derivative appears with respect to fields encoding the $\mu \mathrm{DoF}$. This renders the fluid entropy $S$ a Casimir invariant "of C2 type": the degrees of freedom encoded in $S$ act as "external variables" with respect to the field configuration which would be sufficient to describe the ideal fluid in LF, i.e. the Poisson algebra based on $\vec{\zeta}(\vec{a})$ and $\vec{\pi}(\vec{a})$. Hence, the metric generation of dissipation in this case shows the same mechanism as presented in [20] and in Chapter 8 of [18]. 
Despite the Lagrangian Formulation leads to equations of motion that are more complicated than the ones in EF, stating the dynamics of a viscous fluid in parcel variables appears crucial in order to describe more transparently coherent structures of matter [31].

In their EF, fluids (and plasmas) appear to be often characterised by modes representing local subsets of the continuum in which the parcels move with macroscopic scale correlations (e.g., in vortices or current structures); collective variables describing such field configurations will probably be better described by adopting parcel variables $(\vec{\zeta}, \vec{\pi}, s)$, because long range correlation are likely to form well defined patterns "in the $\vec{a}$-space" rather than "in the $\vec{x}$-space", since the " $\vec{a}$-space" is the set $\mathbf{D}_{0}$ of parcels' identities, where it is possible to keep track of which parcel has interacted with which other one, and hence developed correlation at mesoscopic scales.

Forthcoming studies will investigate the application of what obtained here to the LF of vortices [32], while a contact with the tetrad formalism, describing parcels of various scales [33, 34, 35], will be made.

Last but not least, the LF of an MHD collisional plasma will be constructed, as an extension of the present study to electromagnetic degrees of freedom.

\section{References and Notes}

1. Maupertuis, P. L. Accord de différentes loix de la nature qui avoient jusqu'ici paru incompatibles" (1744).

2. Hamilton, W.R. On a General Method in Dynamics, Philosophical Transaction of the Royal Society, Part I 95 (1835); Part II 247 (1834).

3. Feynman, R. P.; Hibbs, A. R. Quantum Mechanics and Path Integrals, McGraw-Hill Companies; First Edition edition (June 1, 1965).

4. Noether, E. Invariante variationsprobleme, Nachr. Ges. Gottingen, 235 (1918).

5. Goldstein, H. Classical Mechanics, Addison Wesley (2002), ISBN 0-201-65702-3.

6. Hamilton, W. R. On a General Method of Expressing the Paths of Light, and of the Planets, by the Coefficients of a Characteristic Function, Dublin University Review, 795 (1833). Hamilton, W. R. On the Application to Dynamics of a General Mathematical Method previously Applied to Optics, British Association Report, 513 (1834).

7. Arnold, V. I. Mathematical Methods of Classical Mechanics (2nd ed.). New York: Springer (1989). ISBN 978-0-387-96890-2.

8. Dirac, P. A. M. The Principles of Quantum Mechanics, Oxford University Press (1958).

9. Weinberg, S. The Quantum Theory of Fields, Volume 1: Foundations, Cambridge University Press (May 9, 2005), 9780521670531.

10. Tarasov, E. Quantum Mechanics of Non-Hamiltonian and Dissipative Systems, Elsevier Science (08 May 2008), eBook ISBN: 9780080559711, Print Book ISBN: 9780444530912 .

11. Petrovsky, T.; Prigogine, I. The Liouville Space extension of Quantum Mechanics, Advances in Chemical Physics: Resonances, Instability, and Irreversibility, 99, (1997) ISBN 0-471-16526-3, John Wiley \& Sons, Inc. 
12. Gorbatsevich, V. V. On some basic properties of Leibniz algebras, arXiv:1302.3345 [math.RA], Cornell University Library.

13. Guha, P. Metriplectic structure, Leibniz dynamics and dissipative systems, J. Math. Anal. Appl. 326, 121 (2007).

14. Morrison, P. J. Bracket Formulation for Irreversible Classical Fields, Physics Letters A 100, 423 (1984).

15. Morrison, P. J. Some Observations Regarding Brackets and Dissipation, Center for Pure and Applied Mathematics Report PAM-228, University of California, Berkeley (1984).

16. Morrison, P. J. A Paradigm for Joined Hamiltonian and Dissipative Systems, Physica D 18, 410419 (1986).

17. Morrison, P. J. Thoughts on Brackets and Dissipation: Old and New, Journal of Physics: Conference Series 169, 012006 (2009).

18. Fish, D. Metriplectic System, Portland University (2005), PhD Thesis. Downloadable at: http://web.pdx.edu/ djf/.

19. Materassi, M.; Tassi, E. Metriplectic Framework for Dissipative Magneto-Hydrodynamics, Physica D 241, Issue 6, 729 (15 March 2012).

20. Materassi, M.; Tassi, E. Algebrizing friction: a brief look at the Metriplectic Formalism, Intellectual Archive Journal, Toronto: Shiny World (Issue of July 2012).

21. Padhye, N.; Morrison, P. J. Relabeling Symmetries in Hydrodynamics and Magnetohydrodynamics, Plasma Physics Reports 22, 869 (1996).

22. Padhye, N.; Morrison, P. J. Fluid Element Relabeling Symmetry, Physics Letters A 219, 287 (1996).

23. Elskens, Y.; Prigogine, I. From instability to irreversibility, Proc. Nati. Acad. Sci. USA, 83, 5756 (August 1986).

24. Bălescu, R. Statistical Dynamics: Matter Out of Equilibrium, Imperial College Press (1997), ISBN-10: 1860940463.

25. Marsden, J. E.; Morrison, P. J.; Weinstein, A. The Hamiltonian Structure of the BBGKY Heirarchy Equations, Contemporary Mathematics 28, 115 (1984).

26. Bennett, A. F. Lagrangian Fluid Dynamics. Monographs on Mechanics, Cambridge University Press, Cambridge, (2006) ISBN-10: 0521853109.

27. Materassi, M.; Consolini, G.; Tassi, E. Sub-Fluid Models in Dissipative MagnetoHydrodynamics, Magnetohydrodynamics, InTech Publications, Reijka (2012), ISBN 979-953307-381-0.

28. Longhi, G.; Materassi, M. Collective and relative variables for a classical Klein-Gordon field, (1999) International Journal of Modern Physics A, 14 (21), pp. 3387-3420. Lusanna, L.; Materassi, M. A canonical decomposition in collective and relative variables of a Klein-Gordon field in the rest-frame Wigner-covariant instant form", (2000) International Journal of Modern Physics A 15 (18), pp. 2821-2916.

29. Padhye, N. S. Topics in Lagrangian and Hamiltonian fluid dynamics: Relabeling symmetry and ion-acoustic wave stability, dissertation presented to the Faculty of the Graduate School of The 
University of Texas at Austin in Partial Fullment of the Requirements for the Degree of PhD (May 1998).

30. Feynman, R. P. The Feynman Lectures on Physics, Online Edition, 1, § 10, "Conservation of Momentum", website: http://www.feynmanlectures.caltech.edu/I_10.html.

31. Chang, T. Self-organized criticality, multi-fractal spectra, sporadic localized reconnections and intermittent turbulence in the magnetotail, Physics of Plasmas 6, 4137-4145, (1999).

32. Tetrault, D. Turbulent relaxation of magnetic fields, 1. Coarse-grained dissipation and reconnection, J. Geophys. Res. 97, 8531-8540, 1992a. Tetrault, D. Turbulent relaxation of magnetic fields, 2. Self-organization and intermittency, J. Geophys. Res. 97, 8541-8547, 1992b.

33. Vieillefosse, P. Internal motion of a small element of fluid in an inviscid flow, Physica A 125, 150, 1984.

34. Cantwell, B.J. Exact solution of a restricted Euler equation for the velocity gradient tensor, Phys. Fluids A, 4, No. 4, April 1992. Blackburn, H.M.; Mansour, N. N.; Cantwell, B.J. Topology of fine-scales motion in turbulent channel flow, J. Fluid Mech. 310 (1996), 269-292.

35. Chertkov, M.; Pumir, A.; Shraiman, B.I. Lagrangian tetrad dynamics and the phenomenology of turbulence, Physics of Fluids, 05/1999. Naso, A.; Chertkov, M.; Pumir, A. Scale dependence of the coarse-grained velocity derivative tensor: influence of large-scale shear on small-scale turbulence, Journal of Turbulence 7, 41 (2006).

(C) 2014 by the authors; licensee MDPI, Basel, Switzerland. This article is an open access article distributed under the terms and conditions of the Creative Commons Attribution license (http://creativecommons.org/licenses/by/3.0/). 